\title{
THE MULTIPLICITY OF STEADY-STATE SOLUTIONS ARISING FROM MICROWAVE HEATING. I. INFINITE BIOT NUMBER AND SMALL PENETRATION DEPTH
}

\author{
M. I. NELSON ${ }^{1}$, G. C. WAKE ${ }^{2}$, X. D. CHEN $^{3}$ and E. BALAKRISHNAN ${ }^{4}$
}

(Received 7 December, 1998; revised 20 March, 1999)

\begin{abstract}
Microwave heating of porous solid materials has received considerable attention in recent years because of its widespread use in industry. In this study, the microwave power absorption term is modelled as the product of an exponential temperature function with a function that decays exponentially with distance. The latter describes the penetration of the material by the microwaves.

We investigate the phenomena of multiplicity in class A geometries, paying particular attention to how the penetration function affects the behaviour of the system. We explain why the phase-plane techniques which have been used in the case when the penetration term is constant do not extend to non-constant penetration.
\end{abstract}

\section{Introduction}

Microwave technology is being increasingly adopted in industry for heating and/or drying. It has found particular application in the food. industry where it is used for cooking, thawing, freeze drying, pasteurisation, sterilisation, etc. Compared to conventional processes, such as convective heating, its attractions include: that it has brief startup periods; it offers a means of rapid heating; that it is lower in energy consumption, consequently having high energy efficiency; and, that it generally produces a more uniform heating $[20,22,23]$. In some applications, such as in the application of microwaves in reducing surface bacteria counts in cottage cheese [14], highly non-uniform heating is desirable. This can be achieved by increasing the moisture and salt content, reducing the penetration of microwaves through the sample.

\footnotetext{
${ }^{1}$ Department of Fuel and Energy, The University of Leeds, Leeds LS2 9JT, England.

${ }^{2}$ Department of Mathematics and Statistics, University of Canterbury, Private Bag 4800, Christchurch, New Zealand.

${ }^{3}$ Department of Chemical and Materials Engineering, The University of Auckland, New Zealand.

${ }^{4}$ Department of Mathematics and Statistics, Sultan Qaboos University, Sultanate of Oman.

(C) Australian Mathematical Society 2001, Serial-fee code 0334-2700/01
} 
Certain combinations of heating rates and thermophysical properties increase the thermal degradation of materials heated by microwaves, compared to conventional processes [7]. This can lead to thermal runaway, possibly accompanied by increased moisture loss, which adversely affects food properties. Accordingly many studies have been carried out modelling temperature profiles and their thermal stability, with particular attention paid to the formation of hot spots in heated solids $[6,15-18,28]$.

The theoretical work that addresses these issues has recently been reviewed [16]. Although the energy balance for microwave heating is similar to that arising in the theory of thermal explosions, the thermal stability analysis reported in the literature has not taken advantage of the knowledge of self-heating or thermal explosion mechanisms established in the combustion literature.

In this paper, a simple mathematical model for the microwave heating of a regular shaped solid is studied. We approach this problem from the perspective of classical combustion theory.

1.1. A summary of well-known results The main feature of the model we investigate is that the strength of the source term depends not only upon the local temperature but upon the distance from the edge of the sample. In the limit when the dependence upon internal position vanishes we recover the exponential approximation of the Frank-Kamenetskii equations. We therefore review some some salient features of this well-studied system $[2,3,11]$, concentrating on the infinite Biot number case.

The steady-state diagrams for the infinite slab and infinite cylinder contain one limit point. When the Frank-Kamenetskii parameter $(\delta)$ is below criticality, $\delta_{c r}=0.88$ and $\delta_{c r}=2.0$ for the slab and cylinder respectively, there are two steady-state solutions: a 'low-valued' solution that is stable and a 'higher-valued' solution that is unstable. When the Frank-Kamenetskii parameter is larger than criticality, there are no steadystate solutions and the solution blows-up in finite time. For large values of the solution-norm the steady-state locus approaches the $\delta=0$ axis. These classical solution curves are shown as curve (1) in Figure 1 (a)-(b).

The steady-state diagram for the sphere differs from that of the infinite slab or cylinder. For $\delta<1.664$, there is a unique stable solution. For large values of the solution-norm the steady-state curve does not approach the $\delta=0$ axes but instead displays an arbitrarily large number of turning points, with an oscillatory asymptote $\delta \rightarrow \delta_{\infty}=2$ as the solution norm approaches infinity. The smallest (minimal) solution for any $\delta$ is the only stable one. Fujita [12] showed that if there is more than one unstable solution then their spatial profiles intersect in the interior of the domain. When the Frank-Kamenetskii parameter is larger than criticality, $\delta_{c r}=3.32$, there are no steady-state solutions and the solution blows up in finite time. The classical solution curve in the sphere is shown as curve (1) on Figure 4 (a).

The detailed structure of the solutions for the sphere was known in the Russian 


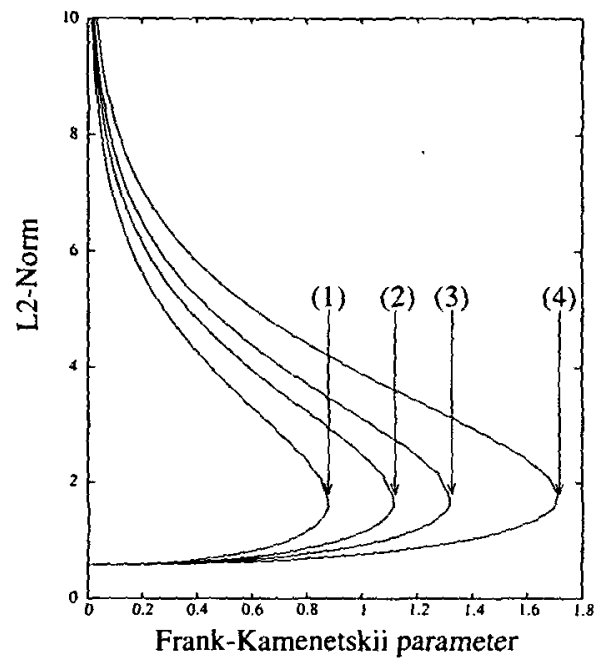

(a) Steady-state diagram for the $\operatorname{slab}(j=0)$.

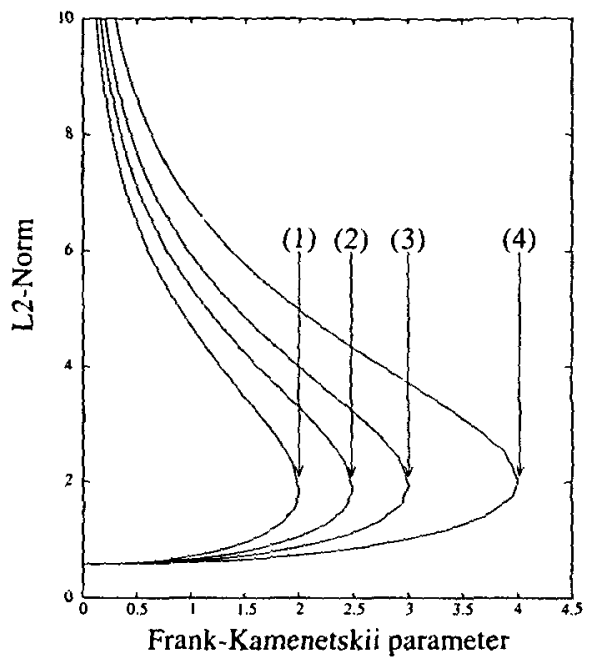

(b) Steady-state diagram for the cylinder $(j=1)$.

Figure 1. In Figure (a) the four curves correspond to: (1) $\beta=0$, (2) $\beta=0.35$, (3) $\beta=0.6$ and (4) $\beta=1.0$. In Figure (b) they correspond to: (1) $\beta=0$, (2) $\beta=0.39$, (3) $\beta=0.74$ and (4) $\beta=1.3$. The dependence of the ignition limit point $\left(\delta_{c r}\right)$ upon the non-dimensionalised decay constant $(\beta)$ is shown in Figure (4) (b).

combustion community by the late 1950's [13], including a proof of infinite multiplicity based upon phase-plane considerations. In the west the phenomena of infinite multiplicity was not recognised until the mid $1960 \mathrm{~s}[10,24]$. The presence of infinite multiplicity was established in the infinite Biot number case by Joseph and Lundgren [19]. They showed that when the shape factor $(j)$ is bounded by $2 \leq j \leq 8$ infinite multiplicity occurs at $\delta_{\infty}=2(j-1)$. This result was extended to finite Biot number by Wake et al. [26], who showed that in the case of the sphere $(j=2)$ infinite multiplicity occurs when $\delta_{\infty}=2 e^{-2 / B i}$, and by Wake and Hood [25], who showed that when $2 \leq j \leq 8$ infinite multiplicity occurs at $\delta_{\infty}=2(j-1) e^{-2 / B i}$. We outline their approach, for infinite Biot number, in Section 3.3 .

\section{Mathematical modelling of microwave heating of a solid material}

Microwave heating is volumetric in nature. When a dielectric sample is exposed to an electromagnetic field, local dipole moments attempt to align with the alternating current field, injecting electrical energy into the dipoles. Intermolecular friction partly dissipates this energy into the medium, causing heating of the sample. The heat generation is usually spatially distributed. 
The internal temperature profile of material that is heated by microwaves is governed by a reaction-diffusion equation in which the 'reaction' term takes the form

$$
\mathscr{R}=q(T)\left\|E_{i}\right\|^{2},
$$

where the function $q(T)$ models the thermal absorption of microwave energy by the sample and $\left\|E_{i}\right\|^{2}$, the square of the electric-field amplitude, represents the penetration of the microwaves into the interior of the sample. The latter quantity is defined by Maxwell's equations, which govern the propagation of electromagnetic radiation in a dielectric medium. In the most general case the dielectric properties of a medium are temperature dependent and so the equations for the spatial variation of the electric and magnetic fields are coupled to a nonlinear heat-conduction equation. The solution of such a system is a formidable task.

In order to present a simplified account of microwave heating we assume that the electric field amplitude decays exponentially with distance. This approximation is discussed further in Sections 2.1-2.2. Other points of interest are discussed in Section 2.3 .

2.1. Electric field intensity The electric field intensity, for waves of a given frequency $\omega$, satisfy

$$
E_{i}=e^{i \omega t} \phi(r),
$$

assuming that its size and the electrical conductivity are independent of temperature. Substitution of (2) into the damped wave equation gives, for the class A geometries of the symmetric infinite slab $(-R<r<R)$, infinite cylinder and sphere

$$
\text { Steady-state equation on } 0<r<R \quad \frac{\mathrm{d}^{2} \phi}{\mathrm{d} r^{2}}+\frac{j}{r} \frac{\mathrm{d} \phi}{\mathrm{d} r}-\frac{\kappa^{2}}{4} \phi=0,
$$

where $r$ is the spatial (radial) co-ordinate in 1 (2 and 3) dimensions respectively, $j=0,1,2$ correspond to the slab, cylinder and sphere respectively, and $\kappa$ is a (possibly complex) constant.

Equation (3) describes the penetration of the electric field through the material. The simplest and most studied case is the slab $(j=0)$ with infinite thickness $(R=\infty)$. In this case

$$
\phi(r)=\phi_{e x r} e^{-\kappa r / 2},
$$

where $\phi_{\text {ext }}$ is the external size of the electric field. The quantity $2 / \kappa$ represents a characteristic thickness of penetration. (Considerable confusion exists with this in the food sciences literature, the omission of the factor 2 for example.) The spatial factor for heating depends on $\|\phi(r)\|^{2}$, which in this case is given by

$$
\exp [-\kappa r] \text {. }
$$


This gives $1 / \kappa$ as the characteristic penetration depth for the power factor. Equation (5) is known as Lambert's law. The application of (5) to materials of finite thickness is discussed further in Section 2.2. However, from a theoretical point of view we should, in the bounded class A geometries, use the solution of (3) with the conditions

$$
\left\{\begin{array}{l}
\phi^{\prime}(0)=0 \quad \text { (symmetry condition) } \\
\phi(R)=\phi_{\text {ex } r}
\end{array}\right.
$$

The solution of (3) with the boundary conditions (6) is

$$
\frac{\phi(r)}{\phi_{e x t}}= \begin{cases}\frac{\cosh (\kappa r / 2)}{\cosh (\kappa R / 2)}, & j=0, \\ \frac{I_{0}(\kappa r / 2)}{I_{0}(\kappa R / 2)}, & j=1, \\ \frac{R \sinh (\kappa r / 2)}{r \sinh (\kappa R / 2)}, & j=2,\end{cases}
$$

where $I_{0}$ is the Bessel function of zeroth order. These solutions (for $j=1,2$ ) show the focusing effect in two and three dimensions. The power term, proportional to $\phi(r)^{2}$, gives the form of the spatial dependence of the heat source term. The form of the various solutions given in (7) are complicated when incorporated in the heat balance equation, but ideally this should be done.

Many authors model the electric field amplitude, in various geometries, by

$$
\phi(r)=\phi_{e x t} e^{\kappa(r-R) / 2} .
$$

This is briefly reviewed in Section 2.2. Note that this approach is compatible with the various forms of (7) when $\kappa$ is large, since in this case we have:

$$
\frac{\phi(r)}{\phi_{e x t}} \begin{cases}=\frac{e^{\kappa r / 2}+e^{-\kappa r / 2}}{e^{\kappa R / 2}+e^{-\kappa R / 2}} \approx e^{\kappa(r-R) / 2}, & \text { for } j=0, \\ \approx \frac{e^{\kappa r / 2} / \sqrt{2 \pi r}}{e^{\kappa R / 2} / \sqrt{2 \pi R}}=\sqrt{\frac{R}{r}} e^{\kappa(r-R) / 2} \approx e^{\kappa(r-R) / 2}, & \text { for } j=1, \\ \approx \frac{R}{r} e^{\kappa(r-R) / 2} \approx e^{\kappa(r-R) / 2}, & \text { for } j=2 .\end{cases}
$$

For the case $j=1$ we have used the asymptotic form of $I_{0}(p)$ for large $p$ and noted that $\phi(r) \approx 0$ except where $R-r$ is small, near the surface. The assumption that $\kappa$ is large implies that the penetration depth is small and that the microwaves are rapidly attenuated.

The outcome of this analysis gives Lambert's law for the electric field intensity $\phi(r)$ in all three geometries under the specified simplifying conditions. 
2.2. Lambert's law The electric field amplitude inside a semi-infinite slab is given by Lambert's law

$$
\left\|E_{i}\right\|^{2}=E_{i, o}^{2} e^{-\kappa(R-r)}
$$

Equation (10) has been widely used to model temperature profiles of materials heated by microwaves $[4,5,9,14,21,27]$ as it represents the simplest possible spatial dependence which has some accepted physical basis, Section 2.1 , but which enables the heating aspects of the problem to be isolated from the electric and magnetic fields. Temperature distributions following exponential decays along the depth have been widely reported. For example, in a recent paper a model based upon Lambert's law adequately predicted temperature distribution for irradiated cottage cheese [14].

Ayappa et al. [1] have shown that Lambert's law holds for a slab of thickness $L(\mathrm{~m})$, heated on two sides, if

$$
L \geq 2\left(\frac{2.7}{\kappa}-0.08\right) \times 10^{-2}
$$

When (11) does not hold, microwave absorption must be calculated from Maxwell's equations.

Curved objects, such as cylindrical and spherical samples, can focus microwaves causing interior points to overheat. In addition the reflection of microwaves from the bottom surface of containers may result in the formation of standing waves, which can cause regional hot and cold spots. As a first approximation we assume that there are conditions under which microwave penetration of such materials can be modelled by what are essentially planar waves and that equations analogous to (11) apply. This approach should apply when the microwaves are rapidly attenuated. ${ }^{1}$

The circumstances under which the power deposition from microwave heating can be approximated by a simple exponential decay in cylindrical samples has been investigated experimentally and numerically by Lobo and Datta [21]. They compared experimental measurements on cylindrical samples of mashed potato with varying salt content to numerical solutions obtained for a semi-infinite slab. Good correlation was found for high values of the decay constant $\kappa$, corresponding to a range of food materials such as water with $1.75 \%$ salt, pork ham and peanut. An exponential decay law was used by Wei et al. [27], who used Lambert's law for the penetration of microwaves into cylindrical water-laden sandstone. Predictions from their model agreed well with observations.

\footnotetext{
'The destruction of standing waves in a finite slab as the attenuation increases has been illustrated by Ayappa $e t$ al. [1]. The thicker the sample the lower the intensity of the reflected wave. Eventually the contribution from the reflected wave is negligible.
} 
2.3. Miscellaneous points We assume that thermal absorption is governed by an exponential dependence,

$$
q(T)=a e^{b T} .
$$

Consequently the reaction term is given by

$$
\mathscr{R}=a E_{i, o}^{2} e^{\kappa(r-R)} e^{b T} .
$$

The product $a E_{i, o}^{2}$ is the rate of heat generation at the microwave surface. The use of a constant decay constant $(\kappa)$ implies that the electrical conductivity is constant; in reality it is temperature dependent with the same dependency as the thermal absorption term, (12). Accurate modelling of this behaviour requires coupling between the equations for the electric field and the temperature and is not considered here.

If significant drying occurs, temperatures in moist foods will tend to reach the boiling point of water and mass transfer must be accounted for. Such behaviour is not considered here. Heat and mass transfer phenomena in microwave heated porous materials have been investigated by Wei et al. [27], based upon Lambert's law.

\section{Model equations}

3.1. Dimensionalised equations The steady-state energy balance for a dry solid undergoing microwave heating is governed by

$$
\begin{aligned}
& \begin{array}{ll}
\begin{array}{l}
\text { Steady-state equation } \\
\text { on } 0 \leq r<R
\end{array} & k\left(\frac{\mathrm{d}^{2} T}{\mathrm{~d} r^{2}}+\frac{j}{r} \frac{\mathrm{d} T}{\mathrm{~d} r}\right)+a E_{i, \omega}^{2} e^{k(r-R)} e^{b T}=0,
\end{array} \\
& \frac{\mathrm{d} T}{\mathrm{~d} r}=0, \quad \text { at } r=0, \\
& T=T_{a}, \quad \text { at } r=R, \quad \text { for infinite Biot number, } \\
& -k \frac{\mathrm{d} T}{\mathrm{~d} r}=h\left(T-T_{a}\right), \quad \text { at } r=R, \quad \text { for finite Biot number. }
\end{aligned}
$$

For simplicity the physical properties are assumed to be constant. When $j=0,1,2$, (14) is the energy balance for an infinite slab, an infinite cylinder and a sphere respectively.

In general this system does not have an analytical solution. However, solutions have been obtained in the special cases $b=0$ [9] and $j=0(b \geq 0$ and arbitrary Biot number) [4].

3.2. Non-dimensionalised equations In non-dimensionalising equations (14)(17) we introduced a non-dimensionalised length, $r^{*}$, and a non-dimensionalised 
temperature, $T^{*}$. The system can then be written as

$$
\begin{aligned}
& \begin{array}{l}
\begin{array}{l}
\text { Steady-state equation } \\
\text { on } 0 \leq r^{*}<1
\end{array} \\
\frac{\mathrm{d}^{2} T^{*}}{\mathrm{~d} r^{* 2}}+\frac{j}{r^{*}} \frac{\mathrm{d} T^{*}}{\mathrm{~d} r^{*}}+\delta e^{\beta\left(r^{*}-1\right)} e^{T^{*}}=0 .
\end{array} \\
& \frac{\mathrm{d} T^{*}}{\mathrm{~d} r^{*}}=0, \quad \text { at } r=0 \text {, } \\
& T^{*}=0, \quad \text { at } r^{*}=1 \text { for infinite Biot number, } \\
& \frac{\mathrm{d} T^{*}}{\mathrm{~d} r^{*}}=-B i T^{*}, \quad \text { at } r^{*}=1 \text { for finite Biot number. }
\end{aligned}
$$

When the non-dimensionalised decay constant, $\beta$, is zero we obtain the well-known Frank-Kamenetskii model $[2,3,11]$. In this paper we consider the infinite Biot number case.

3.3. Reformulation of the problem to allow use of phase plane techniques The existence of infinite multiplicity in high dimensions has been established rigorously using phase plane techniques, Section 1.1. We introduce new variables, $p$ and $q$, and a new length scale, $s$, which acts as a 'time scale', defined by

$$
\begin{aligned}
p & =\delta r^{* 2} e^{-\beta} e^{\beta r^{*}} e^{T^{*}}, \\
q & =r^{*} \frac{\mathrm{d} T^{*}}{\mathrm{~d} r^{*}}+2, \\
r^{*} & =e^{-s}, \quad s>0 .
\end{aligned}
$$

Equation (18) is now written as a system of two non-autonomous equations

$$
\begin{aligned}
& \frac{\mathrm{d} p}{\mathrm{~d} s}=-\beta e^{-s} p-p q, \\
& \frac{\mathrm{d} q}{\mathrm{~d} s}=p+(j-1) q-2(j-1),
\end{aligned}
$$

where $s>0$. When $\beta=0$ this reduces to the model considered by Wake $e$ al. $[25,26]$. Table 1 shows the relationship between the original and new variables. In the phase-plane formulation the problem is to characterise the set of initial conditions which have the property that their trajectory approaches the point $(p, q)=(2,0)$ in the limit $s \rightarrow \infty$.

When $\beta=0(25)-(26)$ have two steady-states, a saddle point $(p, q)=(0,2)$ and an unstable focus $(p, q)=(2,0)$. As $s \rightarrow \infty$ the trajectory that corresponds to a solution of the original partial differential equation enters the saddle point along its stable manifold (see Table 1). It is possible to show that this is a heteroclinic connection from the unstable focus. The number of solutions to the original partial-differential equation is given by the number of intersections of the line $p=\delta$, in the infinite Biot 
TABLE 1. Some properties of (25)-(26) and the related boundary conditions. Note that the initial value of $q$ is unknown, and that solutions of interest have the property that as $s \rightarrow \infty,(p, q) \rightarrow(0,2)$.

\begin{tabular}{|c|cc|}
\hline$r^{*}$ & 0 & 1 \\
\hline $\mathrm{s}$ & $\infty$ & 0 \\
$\mathrm{p}$ & 0 & $\delta$ \\
$\mathrm{q}$ & 2 & $? ? ?$ \\
$T^{*}$ & $T^{*}(0)$ & 0 \\
\hline
\end{tabular}

number case, with this distinguished orbit $[25,26]$. For example, in Figure 7 (a) the line $p=2.05$ has four intersections with the heteroclinic connection-corresponding to four steady-state solutions of the original heat balance equation. When $p=\delta=2$ the straight line passes through the unstable focus and there are an infinite number of intersections-specifying $\delta_{\infty}$. When $\delta$ is large enough the line $p=\delta$ does not intersect the heteroclinic connection and the original pde has no solutions. Hence $\delta_{c r}$ is given by the outer intercept with the heteroclinic connection.

\section{Results}

The steady-state solutions of (18) with boundary conditions (19)-(20) were investigated using Auto 94 [8] by rewriting the initial-value problem as a boundary value problem. Equations (25)-(26) were integrated using a variable-order variable-step method, using the backward differentiation formulae, written to solve stiff problems, which has a high degree of reliability (routine D02EPF in the NAG Fortran Library).

We denote limit points as (L1), (L2), etc., where (L1) is the first limit point to be reached if one traces the steady-state curve, starting at the origin. The limit point (L1) is referred to as the 'ignition limit point'. The notation (CB) refers to a cusp bifurcation.

Note that there is a one-to-one relationship between the non-dimensionalised decay constant $(\beta)$ and the dimensionalised decay constant $(\kappa)$. Hence we often write 'the decay constant' rather than 'the non-dimensionalised decay constant'.

4.1. Steady-state structure Figures 1 and 4 (a) show typical steady-state diagrams for low values of the decay constant for the slab, cylinder and sphere. The steady-state diagrams for the classical combustion problem $(\beta=0)$ are shown for comparison. In all cases the introduction of spatial decay $(\beta \neq 0)$ increases the critical value of the Frank-Kamenetskii parameter, but leaves the basic structure of the solution curves, discussed in Section 1.1, unchanged. In particular, Figure 4 (a) suggests that infinite multiplicity is still exhibited by the sphere for non-zero decay. The critical value of the Frank-Kamenetskii parameter at which infinite multiplicity occurs $\left(\delta_{\infty}(\beta)\right)$ is monotonic in $\beta$. 


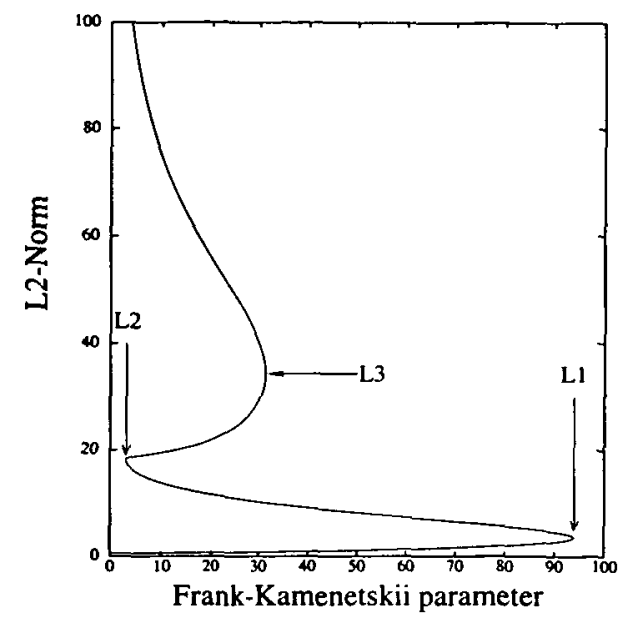

(a) Steady-state diagram for the cylinder $(j=1)$ when $\beta=13$.

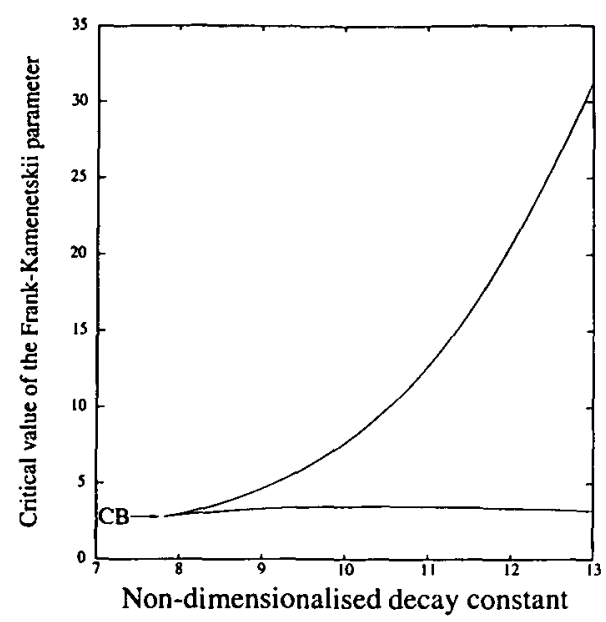

(b) Limit point bifurcation diagram for the cylinder $(j=1)$ when $\beta$ is small.

FIGURE 2. At $\beta \approx 7.82$ there is a cusp bifurcation and when $\beta \geq 7.82$ the steady-state curve for the cylinder has more than one limit point. Compare Figure (a) to Figure 1 (b). In (b) the continuation of the ignition limit point is not shown. The spatial structure of the solutions in Figure (a) when $\delta=20$ is shown in Figure $5(\mathrm{a})$.

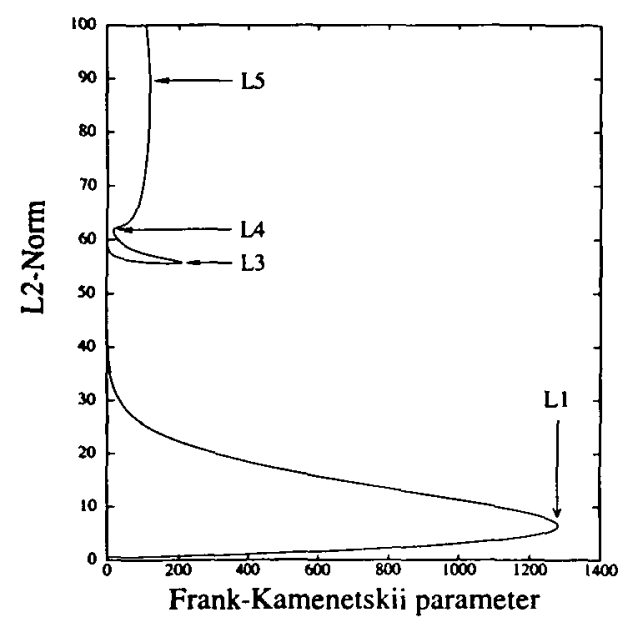

(a) Steady-state diagram for the cylinder $(j=1)$ when $\beta=50$.

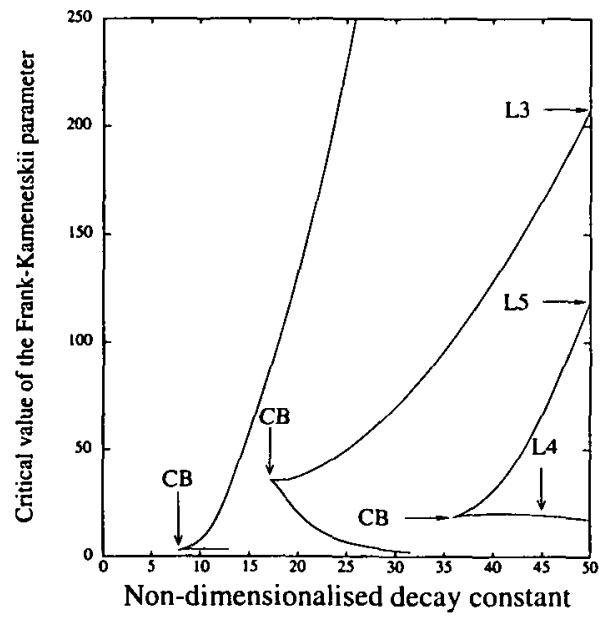

(b) Limit point bifurcation diagram for the cylinder $(j=1)$.

Figure 3. The number of limit points on the steady-state curve for the cylinder increases with increasing B. Compare Figure (a) to Figures I (b) and 2 (a). In (b) the continuation of the ignition limit point is not shown. Note that the abrupt extirpation of two of the the unfoldings in (b) is due to numerical termination and is not a bifurcation. 
Figures 2-3 show that extra structure is found in the case of the cylinder for high values of the decay constant. At $\beta \approx 7.82$ there is a cusp bifurcation, Figure 2 (b), and there is a range of $\beta$ over which the steady-state diagram contains three limit points, illustrated in Figure 2 (a) for the case $\beta=13$. As $\beta$ is increased further there are additional cusp bifurcations and more limit points are introduced into the structure, Figure 3. When $\beta=50$ eleven limit points were found, the first five of which are shown in Figure 3 (a). It is noteworthy that the limit-point curves in Figure 3 (b) are not connected.

Figure 4 (b) shows that the ignition limit-point is monotonic in the non-dimensionalised decay constant.

In Figures 5-6 we investigate the spatial structure of the solutions. Figure 5 (a) shows the spatial structure of the solutions corresponding to a slice through Figure 2 (a) at $\delta=20$. Note that the unstable solutions (B, C and D) intersect each other once in the interior of the domain. Figure 5 (b) shows how the spatial profile of the stable steady-state depends upon the decay constant at a fixed value of the Frank-Kamenetskii parameter. As the decay constant increases the temperature at a fixed point decreases. Furthermore, the boundary region, in which the temperature decreases from an almost constant value to zero, decreases in size as the decay constant increases. High values of the decay constant therefore smooth the internal temperature profile.

Figure 6 shows how the spatial structure of the stable steady-state depends upon the Frank-Kamenetskii parameter at fixed values of the decay constant. These again show a diminishing of the boundary-region at high values of the decay constant.

As the decay constant increases microwave absorption dramatically drops in the interior of the sample. Consequently absorption is concentrated in a decreasing boundary-region near the surface of the sample. For high values of the decay constant transient calculations show that the point at which ignition occurs is near to the surface of the sample. However, when the sample reaches the steady-state solution the maximum temperature will always be in the centre.

4.2. Phase plane techniques In this section we investigate the case $j=2$, corresponding to spherical geometry, using the phase plane formulation of Section 3.3. The case $\beta=0$ is illustrated in Figure 7 (a) and discussed in Section 3.3. When $\beta \neq 0(25)-(26)$ have one steady-state solution, a saddle point, $(p, q)=(0,2)$, and the distinguished orbit that we are interested in reaches it, along its stable manifold, in the limit $s \rightarrow \infty$, Table 1. Although the equilibrium point remains a saddle for all values of time the direction of the linearised stable manifold now depends upon time.

In the absence of a heteroclinic connection it is natural to ask if there is a homoclinic orbit to the saddle point. This possibility can be eliminated by considering (25)-(26) in conjunction with Figure 7 (b). Note that the line $p=0$ is invariant, that the equilibrium point is on the line $p=0$, and that if $p>0$ and $q>0$ then $\mathrm{d} p / \mathrm{d} s<0$. 


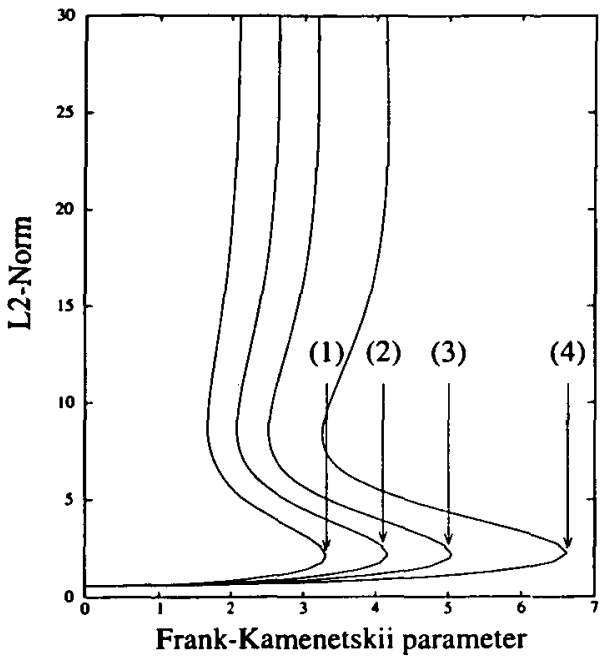

(a) Steady-state diagram for the sphere $(j=2)$.

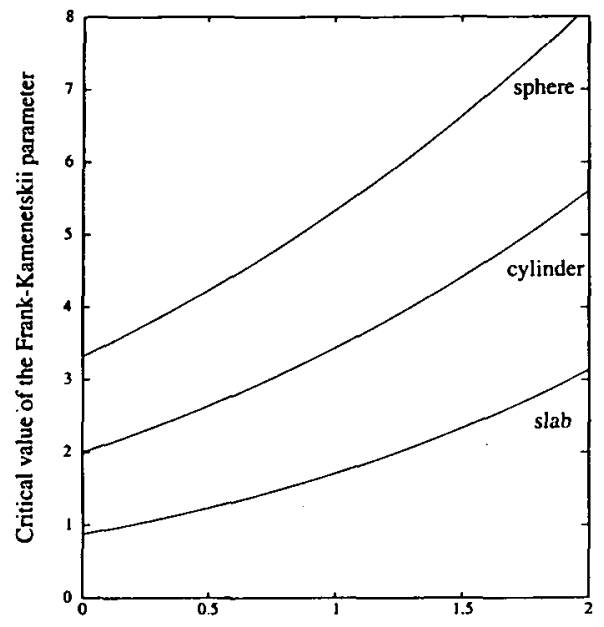

Non-dimensionalised decay constant

(b) The dependence of the ignition limit point $\left(\delta_{c r}\right)$ with non-dimensionalised decayconstant $(\beta)$.

Figure 4. In Figure (a) the four curves correspond to: (1) $\beta=0$, (2) $\beta=0.47$, (3) $\beta=0.88$ and (4) $\beta=1.5$.

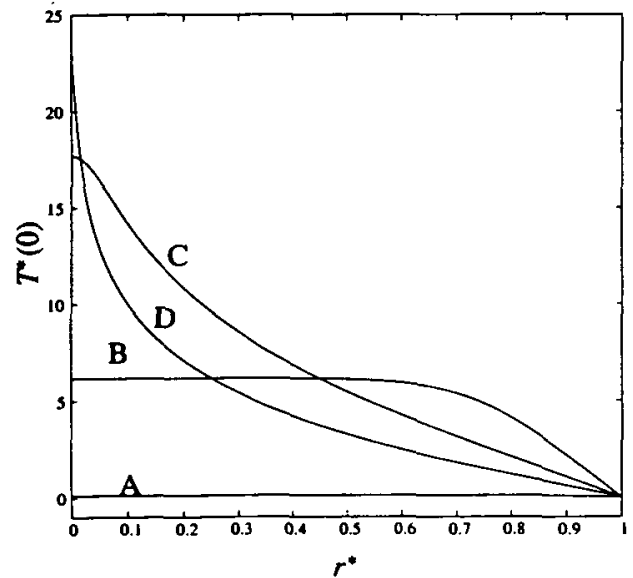

(a) The four steady-state profiles when $\beta=$ 13 and $\delta=20$. Only the lowest profile (A) is stable.

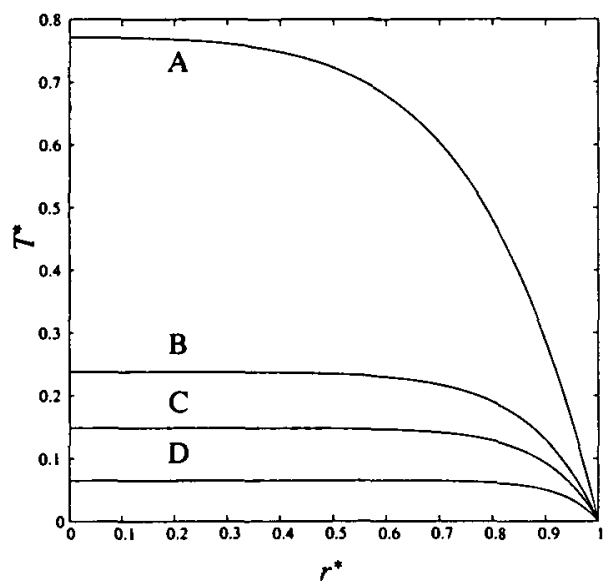

(b) The variation of temperature profiles with $\beta$ for fixed $\delta$.

Figure 5. Parameter value common to (a) and (b): $j=1$. Parameter values in (b): $\delta=15$; (A) $\beta=4$, (B) $\beta=8$, (C) $\beta=10$, (D) $\beta=15$. See Figure 2 (a) for the steady-state structure when $\beta=13$. 


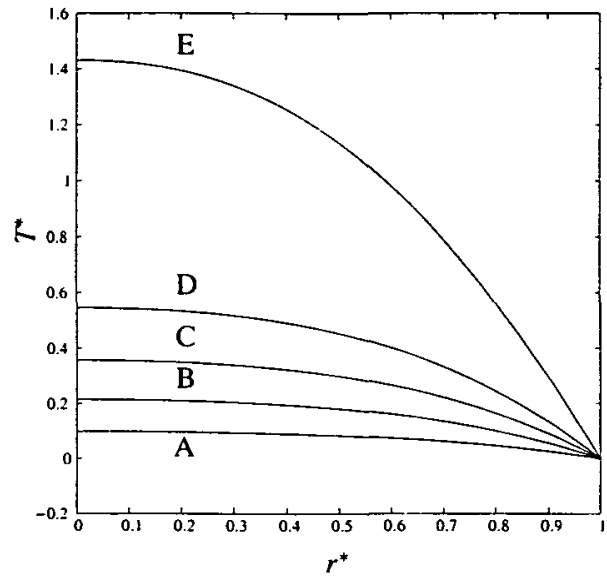

(a) The dependence of the temperature profile upon $\delta$ for fixed $\beta$ ( $\beta$ low) in a cylinder $(j=1)$.

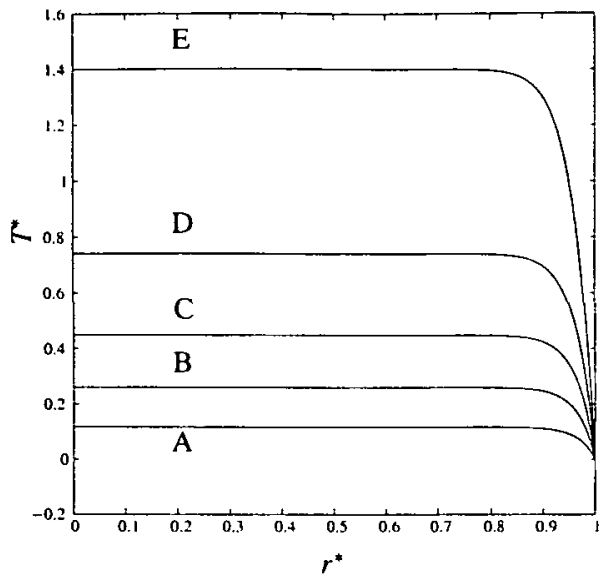

(b) The dependence of the temperature profile upon $\delta$ for fixed $\beta$ ( $\beta$ high) in a cylinder $(j=1)$.

FIGURE 6. Parameter values in (a): $\beta=2$; (A) $\delta=2$, (B) $\delta=3$, (C) $\delta=4$, (D) $\delta=\delta_{\text {cr }}=5.5972$. Parameter values in (b): $\beta=30$; (A) $\delta=200$, (B) $\delta=300$, (C) $\delta=400$, (D) $\delta=\delta_{\text {cr }}=469.73$.

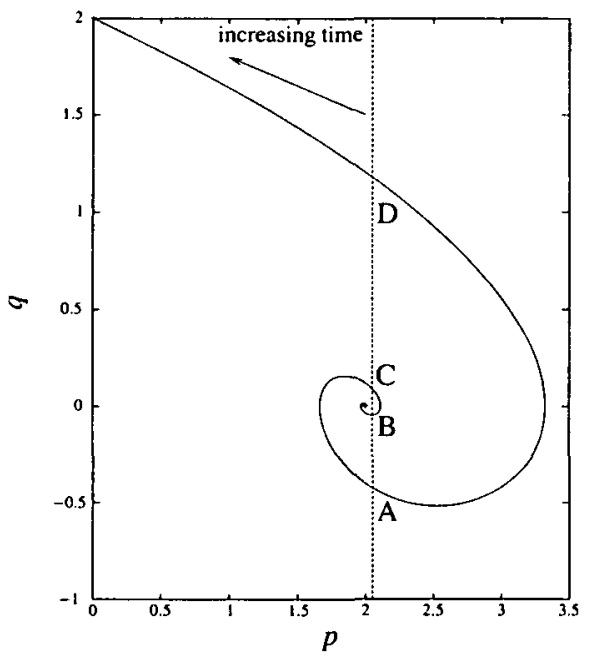

(a) The heteroclinic connection between the equilibrium points $(2,0)$ and $(0,2)$ when $\beta=0$.

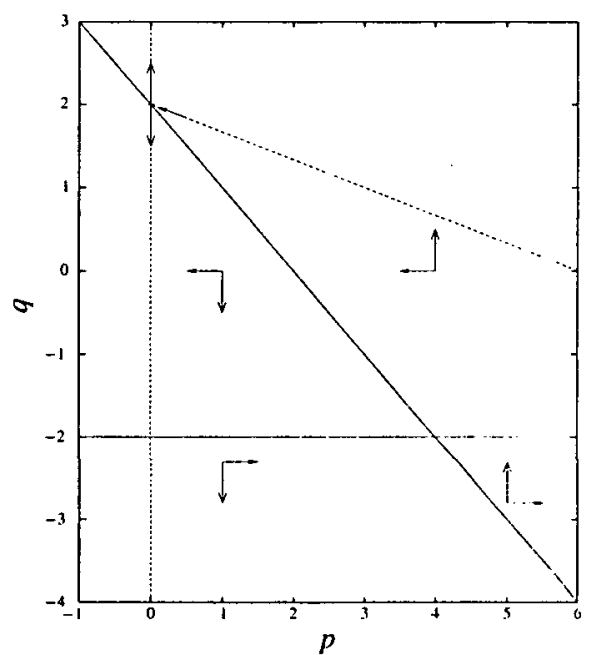

(b) Phase plane analysis in the case $\beta \neq 0$.

FIGURE 7. Phase-plane analysis: (a) When $\beta=0$ and $\delta=2.05$ there are four steady-state solutions, corresponding to an initial condition given by one of $\mathrm{A}, \mathrm{B}, \mathrm{C}$ or $\mathrm{D}$ at $s=0$, with evolution to the steady-state $(0,2)$ as $s \rightarrow \infty$; (b) The null-cline $q=2-p$ and the pseudo null-cline $q=-\beta e^{-s}$ (with $\beta e^{-s} 2$ ) are heavy lines. The dashed lines show three of the linearised eigenvectors. The arrows show the direction in which the components of the vector field point. Parameter values: $j=2$. 


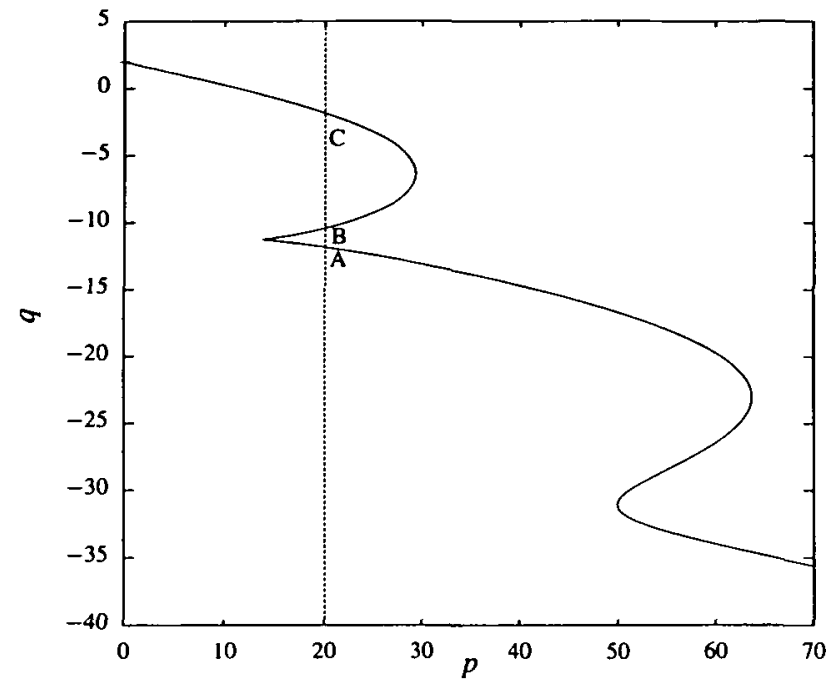

FIGURE 8. One of the trajectories entering the saddle-point in the non-autonomous system $(\beta=0.05)$.

Consequently in the vicinity of the equilibrium point all trajectories with $p>0$ have $p$ decreasing, so no trajectory can emerge from the equilibrium except for those on the invariant line $p=0$.

Where does the trajectory that enters the equilibrium point in the limit of infinite time originate from? In the absence of a homoclinic orbit and in view of the vector field sketched in Figure 7 (b) it must originate at $(+\infty,-\infty)$. Thus the line $p=\delta$ always intersects the distinguished orbit, suggesting that there is always at least one steadystate solution to the original problem. This interpretation contradicts Figure 4 (a).

However, in the non-autonomous case intersections of the line $p=\delta$ with the distinguished orbit are not guaranteed to reach the saddle point as $s \rightarrow \infty$ if the integrations are started at $s=0$. The intersection argument works in the autonomous case because the equations are invariant under translation of time: any initial condition, with $s=0$, on the heteroclinic connection reaches the saddle point at infinite time. Originally, we thought that this would carry over to the non-autonomous case.

To show that this is not the case consider Figure 8 and assume that point $\mathrm{C}$ is an initial condition $(s=0)$ whose trajectory enters the saddle point after infinite time. Suppose that the points $\mathrm{B}$ and $\mathrm{A}$ are reached by integrating the equations backwards from $C$ after 5 and 10 units of time respectively. In the autonomous case, Figure 7 (a), points $B$ and $A$ were guaranteed to reach the saddle point if they were used as initial conditions with time set to zero. However, this is not true in the non-autonomous case. If we start at B with time $s=-5$ then we reach the saddle point, but if we start at B with time $s=0$ this is not guaranteed: the non-autonomous equations are not time-invariant and the vector field depends upon the time value. 
TABLE 2. Comparison of the exact value of $\delta_{c r}$ with the bound in (29).

\begin{tabular}{|c|c|c|}
\hline$\beta$ & $\delta_{c r}(\beta)$ & $\delta_{c r, F K} e^{\beta}$ \\
\hline 0 & 3.322 & 3.322 \\
0.47 & 4.178 & $3.322 e^{0.47}=5.315$ \\
0.88 & 5.054 & $3.322 e^{0.88}=8.009$ \\
1.50 & 6.629 & $3.322 e^{1.50}=14.888$ \\
\hline
\end{tabular}

For the non-autonomous case there is no guarantee that there is a unique trajectory that enters the saddle point as $s \rightarrow \infty$. Furthermore, we need to know exactly the initial condition corresponding to $s=0$, we cannot choose an arbitrary point on the trajectory. Figure 4 (a) suggests that the introduction of the penetration function does not destroy the phenomena of infinite multiplicity. A new idea is therefore required to characterise the set of initial conditions whose trajectories enter the saddle point.

\section{Discussion}

By comparing (18) with

$$
\frac{\mathrm{d}^{2} V}{\mathrm{~d} r^{* 2}}+\frac{j}{r^{*}} \frac{\mathrm{d} V}{\mathrm{~d} r^{*}}+\delta e^{-\beta} e^{V}=0, \quad r^{*} \in(0,1),
$$

we have, since $\delta e^{-\beta}<\delta e^{\beta\left(r^{*}-1\right)}$ when $r^{*} \in(0,1)$, that $V$ is a lower solution for (18) with the same boundary conditions.

Clearly, for this problem, we have criticality when

$$
\delta_{c r} e^{-\beta}=\delta_{c r, F K},
$$

where $\delta_{c r, F K}$ is the limit point bifurcation value for the Frank-Kamenetskii problem: $\delta_{c r, F K}=0.88,2.00,3.32$ for $j=0,1,2$ respectively.

As $V$ is a lower solution, the true critical value for (18)-(21) will be lower than for (27) (the true limit point is lower) and so

$$
\delta_{c r, F K} e^{\beta}=\delta_{c r}>\delta_{c r}(\beta) .
$$

This provides a bound and shows that there are no solutions for $\delta$ large and $\beta \geq 0$. This is a useful result to keep in mind when considering the non-autonomous phase-plane results of Section 4.2. For $j=2$ we have the results shown in Table 2.

\section{Conclusion}

We have investigated a simple model for the microwave heating of dry materials in class $\mathrm{A}$ geometries in which the classical Frank-Kamenetskii reaction term is 
multiplied by a term modelling the spatial penetration of the body by the microwaves. The critical value of the Frank-Kamenetskii parameter increases monotonically with the decay constant.

Although there are many similarities with the classical theory of thermal explosion high values of the decay constant introduce additional multiplicity in the case of an infinite cylinder. For the case of the sphere we have shown that previously-used phase-plane techniques do not work: the incorporation of a term modelling spatial penetration renders the equations non-autonomous and a new method is required to identify the point of infinite multiplicity.

\section{Acknowledgements}

This work was carried out whilst MIN was supported by a fellowship from the Royal Society of London to work in the Department of Mathematics and Statistics at the University of Canterbury (New Zealand).

\section{References}

[1] K. G. Ayappa, H. T. Davis, G. Crapiste, E. A. Davis and J. Gordon, "Microwave heating: An evaluation of power formulations", Chem. Eng. Sci. 46 (1991) 1005-1016.

[2] T. Boddington, P. Gray and G. C. Wake, "Criteria for thermal explosion with and without reactant consumption", Proc. Roy. Soc. A 357 (1977) 403-422.

[3] P. C. Bowes, Self-heating: evaluating and controlling the hazards (Elsevier, Amsterdam, 1984).

[4] X. D. Chen, "Microwave heating in an infinite solid slab and its thermal stability analysis using steady state bifurcation theory", J. Food Eng. 35 (1998) 339-349.

[5] C. J. Coleman, "The microwave heating of frozen food substances", Appl. Math. Modelling 14 (1990) 439-443.

[6] C. J. Coleman, "On the microwave hot spot problem", J. Austral. Math. Soc. Series B 33 (1991) 1-8.

[7] A. K. Datta and J. Liu, "Thermal time distributions in microwave and conventional heating of food", Trans. Inst. Chem. Engnrs. 70C (1992) 83-90.

[8] E. Doedel, X. Wang and T. Fairgrieve, AUTO 94 Software for Continuation and Bifurcation Problems in Ordinary Differential Equations, November 1994, Applied Mathematics Report California Institute of Technology Pasadena, California 91125.

[9] J. Dolande and A. Datta, "Temperature profiles in microwave heating of solids: systematic study", J. Microwave Power and Electromagnetic Energy 28 (1993) 58-67.

[10] J. W. Enig, "Critical parameters in the Poisson-Boltzmann equation of steady-state thermal explosion theory", Combustion and Flame 10 (1966) 197-199.

[11] D. A. Frank-Kamenetskii, Diffusion and heat transfer in chemical kinetics, 2nd ed. (New York, Plenum Press, 1969).

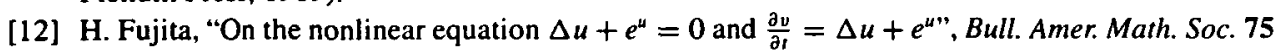
(1969) 132-135. 
[13] I. M. Gel'fand, "Some problems in the theory of quasilinear equations", Uspehi Mat. Nauk 14 (1959) 87-158, English translation, Amer. Math. Soc. Translations 29, 295-381, 1963.

[14] A.-G. Herve, J. Tang, L. Luedecke and H. Feng, "Dielectric properties of cottage cheese and surface treatment using microwaves", J. Food Eng. 37 (1998) 389-410.

[15] J. M. Hill, "Simple exact solutions applicable to microwave heating", J. Appl. Math. Phys. (ZAMP) 40 (1989) 872-882.

[16] J. M. Hill and T. R. Marchant, "Modelling microwave heating", Appl. Math. Modelling 20 (1996) $3-15$.

[17] J. M. Hill and A. H. Pincombe, "Some similarity profiles for the microwave heating of a half-space", J. Austral. Math. Soc. Series B 33 (1991) 29-30.

[18] J. M. Hill and N. F. Smyth, "On the mathematical analysis of hot spots arising from microwave heating", Math. Eng. in Industry 17 (1990) 1823-1834.

[19] D. D. Joseph and T. S. Lundgren, "Quasilinear Dirichlet problems driven by positive sources", Archive for Rational Mech. and Anal. 49 (1972-73) 241-269.

[20] Y. E. Lin, R. C. Anantheswaran and V. M. Puri, "Finite element analysis of microwave heating of solid foods", J. Food Eng. 25 (1995) 85-112.

[21] S. Lobo and A. K. Datta, "Characterization of spatial non-uniformity in microwave reheating of high loss foods", J. Microwave Power and Electromagnetic Energy 33 (1998) 158-166.

[22] D. G. Prabhanjan, H. S. Ramaswamy and G. S. Raghavan, "Microwave-assisted convective air drying of thin layer carrots", J. Food Eng. 25 (1995) 283-293.

[23] S. Ryynanen, "The electromagnetic properties of food materials: a review of the basic principles", J. Food Eng. 26 (1995) 409-429.

[24] J. J. Steggerda, "Thermal stability: An extension of Frank-Kamenetsky's theory", J. Chem. Phys. 43 (1965) 4446-4448.

[25] G. C. Wake and M. J. Hood, "Multiplicity of solutions of a quasilinear elliptic equation in spherical domains", Math. Comput. Modeling 18 (1993) 157-162.

[26] G. C. Wake, S. K. Scott and J. Brindley, "Multiplicity of steady state solutions of combustion in a sphere with arbitrary Biot number", Phys. Lett. 153A (1991) 16-21.

[27] Chen Kou Wei, H. T. Davis, E. A. Davis and J. Gordon, "Heat and mass transfer in water-laden sandstone: Microwave heating", Amer. Inst. Chem. Eng. J. 31 (1985) 842-848.

[28] S. Zhu, Y. Zhang and T. Marchant, "A DRBEM model for microwave heating problems", Appl. Math. Modelling 19 (1995) 287-297. 